\title{
Auditory Brainstem Implantation in Children
}

\author{
Levent Sennaroglu • Gonca Sennaroglu • \\ Gamze Atay
}

Published online: 17 April 2013

(C) Springer Science+Business Media New York 2013

\begin{abstract}
Until last decade, use of auditory brainstem implant (ABI) was restricted to adults and children with severe inner ear malformations and cochlear nerve aplasia did not have any option for hearing rehabilitation. Use of ABI opened a new era for this group of patients. In this review article, definite and probable indications of $\mathrm{ABI}$ for children are stated. Among probable indications, children with hypoplastic nerves constitute the most challenging group in decision making between cochlear implant (CI) and ABI. In these patients, radiological findings should be utilized together with preoperative and intraoperative audiological evaluation. However, none of these assessment methods offer enough data to enable correct choice between $\mathrm{CI}$ and ABI. Intracochlear electrical ABR (eABR) seems to a better indicator compared to preoperative electrophysiological tests. Commonly, CI is the first choice in these patients; if benefit with CI is not satisfactory during follow up, then ABI is utilized. Further definitive diagnostic tools are require in order to overcome this uncertainty. The ABI team must be experienced on pediatric CI patients, also an experienced pediatric neurosurgeon is indispensable to achieve success and to avoid complications. Age limit for ABI is similar to CI candidate children. Better language outcome is expected at younger ages, i.e., between 1 and 2 years. Preoperative radiological work up involves both high resolution computed tomography and magnetic resonance imaging. The side with less
\end{abstract}

L. Sennaroglu $(\bowtie) \cdot$ G. Atay

Department of Otolaryngology, Hacettepe University Medical

Faculty, Ankara, Turkey

e-mail: lsennaroglu@gmail.com

G. Sennaroglu

Department of Otolaryngology, Audiology Unit, Hacettepe

University Medical Faculty, Ankara, Turkey severe inner ear malformation or with more developed neural structures can be preferred. In preoperative audiological evaluation, subjective tests should always be included in test battery as well as objective ones. Rehabilitative assessment is focused on auditory perception skills, language and speech skills and learning abilities. Surgical method of choice is retrosigmoid approach in children. Intraoperative eABR measurements are important to determine correct position of electrode. Interindividual variability in language development and audiological outcome is prominent. Overall complication rate is in an acceptable range when surgery is performed with an experienced team. In conclusion, ABI can provide satisfactory audiological outcomes and language development to a certain extent is possible when every aspect of whole process is applied properly.

Keywords Auditory brainstem implant - Children . Inner ear malformation

\section{Introduction}

In 2001 Colletti et al. [1] reported their auditory brainstem implant (ABI) experience in two children with severe inner ear malformations and no apparent cochlear nerve, for the first time in literature, 2 years after the surgery. Until that time, cochlear implant (CI) surgery was contraindicated in these patients and no appropriate rehabilitation was possible. Use of ABI for children opened a new era in the rehabilitation of patients, in whom CI surgery was contraindicated due to cochlear, labyrinthine or cochlear nerve aplasia. In their initial paper, they reported that both patients had achieved good environmental sound awareness and some speech detection. After a period of time, other 
centers also started to use ABI for rehabilitation of these children.

\section{Indications}

ABI can be used in children with severe malformations and complete ossification of cochlea after meningitis. Inner ear malformations constitute the main group. ABI is not required in all cochleovestibular malformations. Patients with incomplete partition type II and III, and large vestibular aqueduct almost always have cochlear development to a certain extent and therefore they can be rehabilitated with CI. In the consensus paper, Sennaroglu et al. [2] divided the indications into two groups:

\section{A Definite indications}

(1) Complete labyrinthine aplasia (Michel aplasia). The cochlea, vestibule, vestibular aqueduct and cochlear aqueduct are absent (Fig. 1).

(2) Cochlear aplasia. This is absence of the cochlea. The accompanying vestibular system may be normal (Figs. 2a) or there may be an enlarged vestibule (Fig. 2b).

(3) Cochlear nerve aplasia. This is the absence of the cochlear nerve (Fig. 3).

(4) Cochlear aperture aplasia. This is the absence of the bony channel transmitting the cochlear nerve from the IAC to the cochlea. (Fig. 4).

B Probable indications

(1) Hypoplastic cochlea with hypoplastic cochlear aperture. Hypoplastic cochleae may have different audiological presentation. Some patients may be aided with hearing aids and they may have excellent speech and language development. If they are accompanied by hypoplastic

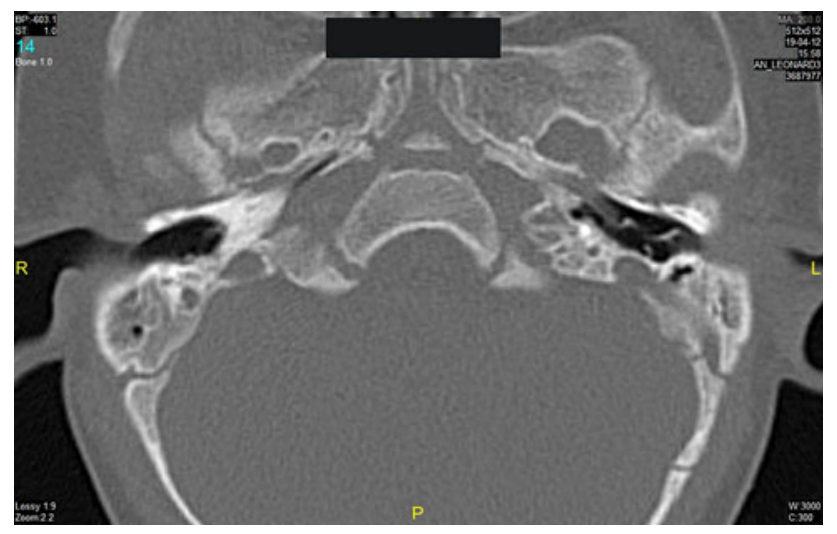

Fig. 1 Michel deformity where the cochlea, vestibule, vestibular aqueduct and cochlear aqueduct are absent cochlear aperture on high resolution computed tomography (HRCT), usually cochlear nerve is hypoplastic or absent and they commonly have severe to profound hearing loss (Fig. 5a, b). In the latter group, the cochlear nerve entering the cochlea is hypoplastic and it is difficult to determine accurately the functional capacity of the cochlear nerve with the present tests.

(2) Common cavity and incomplete partition type I cases where cochlear nerve is apparently missing. If the cochlear nerve is present they are candidates for cochlear implantation. It is important to note that common cavity can be easily confused with cochlear aplasia and vestibular dilatation. The results of $\mathrm{CI}$ in cochlear aplasia and vestibular dilatation are not successful and this should be avoided [3•].

(3) Common cavity and incomplete partition type I cases if the cochlear nerve is present. Even if the nerve is present, the distribution of the neural tissue in the abnormal cochlea is unpredictable, and ABI may be indicated in such cases if $\mathrm{CI}$ fails to elicit an auditory sensation.

(4) The presence of an unbranched cochleovestibular nerve $(C V N)$ is a challenge in these cases. In this situation, it is not possible to determine the amount of cochlear fibers travelling in the CVN. If there is a suspicion, a CI can be used in the first instance, and ABI can be reserved for the patients in whom there is insufficient progress with $\mathrm{CI}$.

(5) The hypoplastic cochlear nerve presents a dilemma for the implant team. A hypoplastic nerve is defined as less than $50 \%$ of the usual size of the cochlear nerve or less than the diameter of the facial nerve. Radiology of these patients should be carefully reviewed with an experienced neuroradiologist. If sufficient amount of neural tissue cannot be followed into the cochlear space, an ABI may be indicated.

Children with hypoplastic nerves or thin unbranched CVN constitute the most controversial group in decision making between CI and ABI. It must be kept in mind that, children with hypoplastic nerves usually do not reach levels of those with normal cochleae, in terms of hearing and language development. It is obvious that, radiology may not predict the presence of the cochlear nerve accurately in these mentioned challenging five group of patients. In all these subjects audiological findings, as well as radiological findings, should be used together in order to decide between $\mathrm{CI}$ and ABI. If an experienced pediatric audiologist detects a slight response on either side of 
Fig. 2 Cochlear aplasia with normal vestibular system (a) and an enlarged vestibule (b) (a)

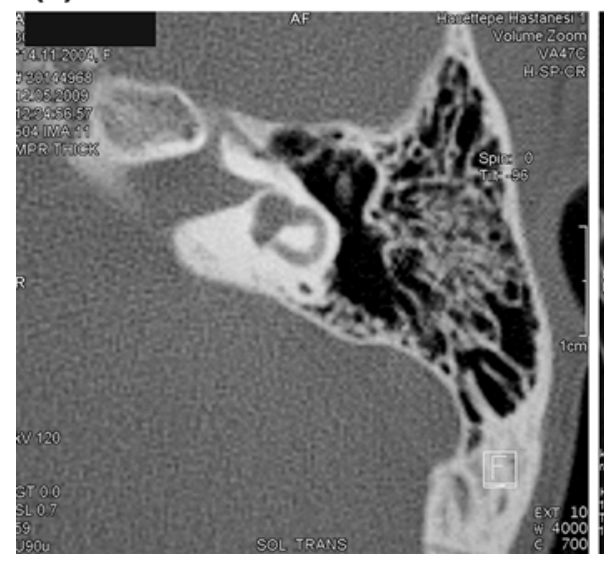

(b)

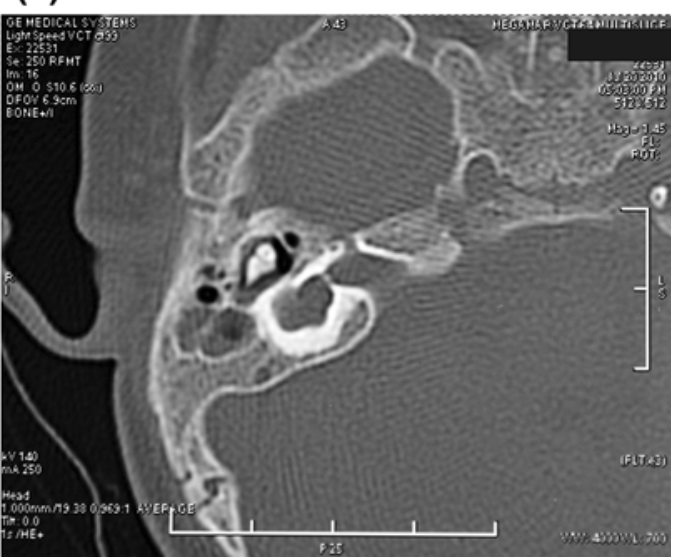

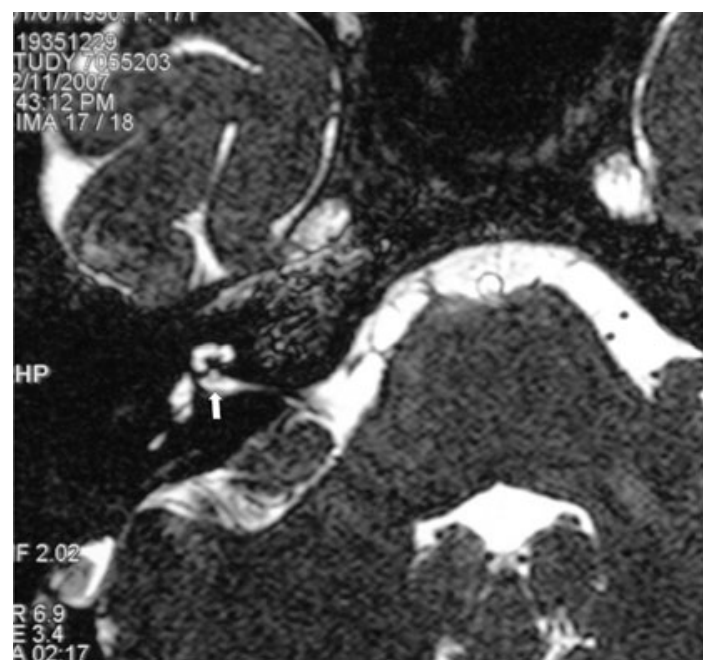

Fig. 3 Cochlear nerve aplasia. MRI showing no cochlear nerve going towards the hypoplastic cochlea (white arrow)

these cases, this information is very valuable in the side selection of CI. In such cases, family should be carefully counseled about the possibility of ABI surgery if insufficient progress with $\mathrm{CI}$ is encountered during postoperative follow up.

Some cases of pneumococcal meningitis produce total cochlear ossification (Fig. 6) where a CI cannot be placed satisfactorily into the scala timpani. Different surgical techniques (such as drill-out cochleostomy) have been described and electrode options (double or split array implants) are produced for total ossification. These usually result in suboptimal results. ABI is another option for patients with total ossification because the electrode can be placed in a normal location. In partial ossification however, every effort should be done to place the electrode in the scala tympani or vestibuli. If the electrode is not satisfactorily placed into either scala tympani or scala vestibuli and ABI may be another option.

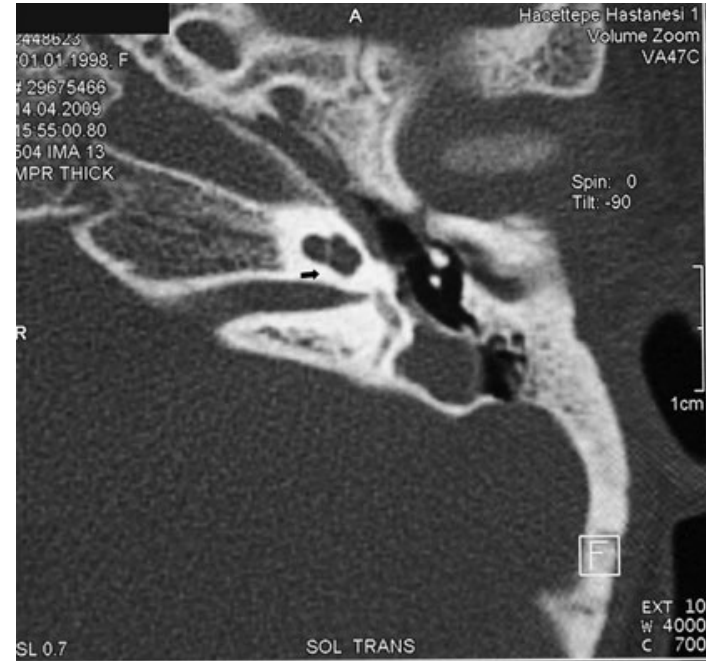

Fig. 4 Cochlear aperture aplasia: The bony channel transmitting the cochlear nerve from the IAC to the cochlea is absent (black arrow)

\section{Versus ABI in Children with Hypoplastic Cochlear Nerve}

There is a controversy regarding the type of implant to be used in the treatment of patients with hypoplastic cochlear nerve. Although it is possible to obtain good hearing and language development in certain cochlear hypoplasia with hypoplastic cochlear nerve, some have insufficient or no hearing, and limited language development with CI. These patients become candidates for ABI. It is important to correctly diagnose this subset of children and proceed with $\mathrm{ABI}$ directly when required; however, for the present time, preoperative and intraoperative audiological tests are not precise enough to enable correct diagnosis.

Warren et al. [4] reported three cases with narrow internal auditory canals baring two nerves, one of each seemed to enter vestibule in each case and the other was assumed to be the functioning facial nerve. Two of the 
Fig. 5 Hypoplastic cochlear aperture (a) (black arrow) with a hypoplastic cochlear nerve (b) (black arrow) (a)

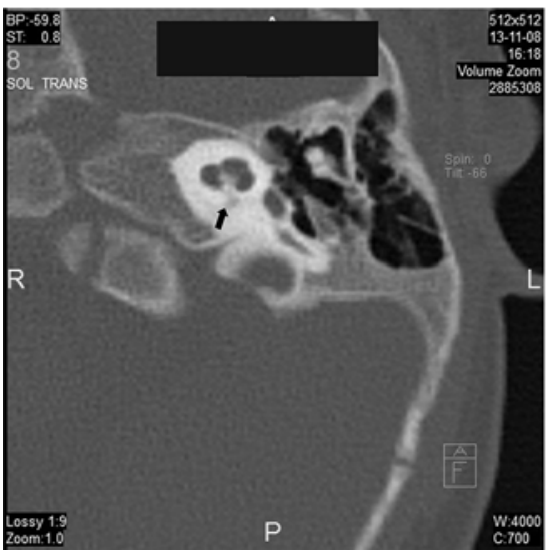

(b)

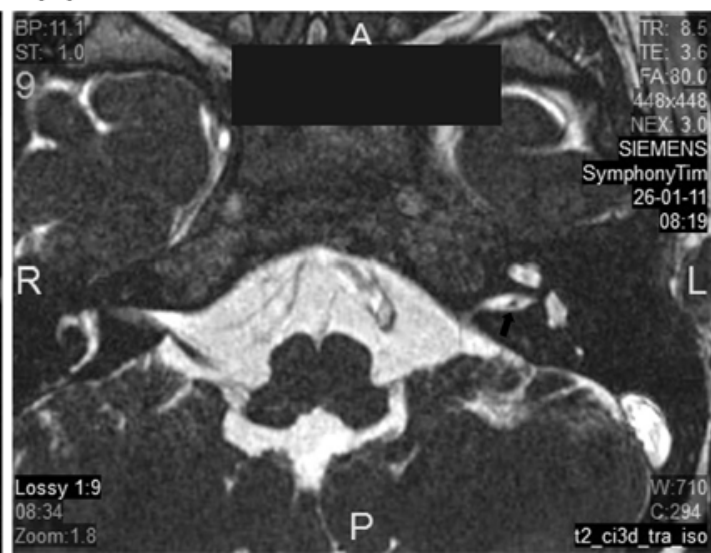

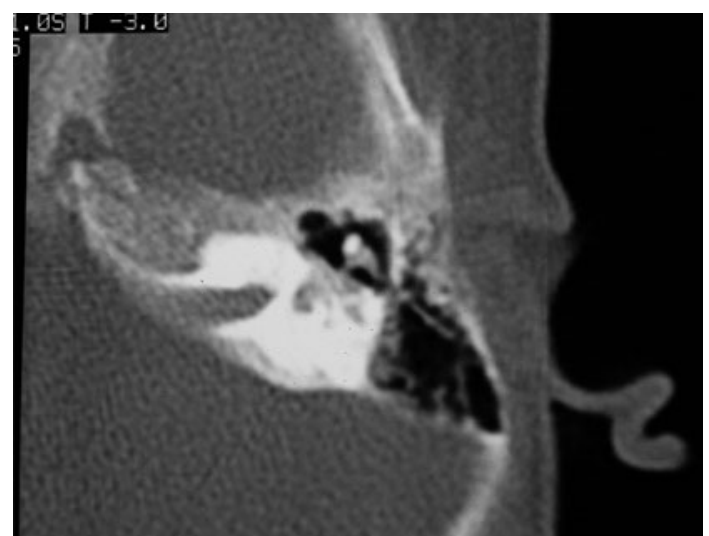

Fig. 6 Severe cochlear ossification

families reported responses to auditory stimuli with amplification over time. Promontory stimulation testing yielded reproducible responses to electrical stimuli in the ears of question. They all underwent cochlear implantation. Early results after CI (4, 5 and 9 months, respectively) showed responses to auditory stimuli. They tried to explain the mechanism of sound transmission despite the fact that there were no visible cochlear branches on MRI and proposed two main possibilities. Either there was a very tiny branch which could not be visualized due to extremely narrow distal IAC or the nerve enters the vestibule and then turns toward cochlea. Regarding similar cases, our group observed that progress usually reaches a level, and language development usually does not gain the level of CI in normal cochlea. These children usually obtain certain hearing levels after $\mathrm{CI}$ but this may not be usually sufficient for appropriate language development.

Valero et al. [5*0] recorded electrically evoked responses in the majority of CI recipients with hypoplasia of the auditory nerve (AN). The atypical amplitude and latencies of these responses suggested nonauditory generators and should not be misread as typical EABR peaks. Chronic CI stimulation of the hypoplastic nerve did not promote normal auditory brainstem maturity and did not discourage uncharacteristic development in the brainstem. The type of abnormal evoked response cannot be predicted by the severity of the hypoplastic AN, auditory nerve canal (ANC), or IAC; there was no relationship between auditory pathway size and evoked brainstem response to determine whether they will be good CI candidates with these structural defects, but the unpredictable evoked responses observed here would make it difficult to predict auditory outcomes. Although there was limited initial improvement in speech perception outcomes, children with stenotic IAC and hypoplastic AN did not achieve comparable behavioral results with their CIs compared with children with an uncompromised AN. Moreover, the difference in terms of behavioral tests were kept even in long term follow up, i.e., children with compromised AN were found to be at scores of 24 months of those with normal AN at their 120th follow-up month. These data, along with abnormal electrophysiological findings, suggest that children with hypoplasia of the AN, are not stellar candidates for cochlear implantation. If the decision is made to proceed with cochlear implantation, families should be counseled that expectations of auditory and spoken language development should be tempered.

Buchman et al. [6] reported CI results in patients with labyrinthine anomalies. They concluded that the implanted children with cochlear nerve deficiency had poor speech perception abilities. This suggested that the peripheral neural populations in patients with cochlear nerve deficiency are insufficient for the development of synchronized auditory stimulation in most instances. They proposed initial usage of CI before ABI in these situations. One of the important findings of this study was that, intracochlear eight nerve compound action potential (ECAP) testing results were associated with the development of speech perception abilities. However, they emphasized the 
requirement of further electrical stimulation technologies prior to placement of CI.

Song et al. [7] reported their results of intracochlear evoked auditory brainstem response (EABR) versus extracochlear EABR in predicting long-term outcomes of patients with narrow IAC. They concluded that intracochlear EABR measured either intraoperatively or in the early postoperative period may play an important role in deciding whether to continue with auditory rehabilitation with a CI or to switch to an ABI so as not to miss the optimal timing for language development. They also compared evoked compound action potential (ECAP) and EABR measurements and found that in cases with inner ear anomalies, including narrow IAC where the number of AN fibers that can be stimulated is limited, intracochlear EABR can be more successfully recorded than can ECAP. They concluded that for these cases in which cochlear implantation has been performed initially, considering the limited prognostic value of preoperative extracochlear electrophysiologic testing or imaging, intracochlear EABR measured either intraoperatively or in the early postoperative period may provide valuable prognostic information to predict long-term outcomes.

Song et al. [8] argued that promontory stimulation test may not predict the long-term outcome accurately in cases with hypoplastic cochlear nerves. They correlated the diameter of IAC on HRCT with the presence of CVN during surgery. These findings suggest that the presence or absence of the CVN could not be accurately predicted by the diameter of the IAC measured on temporal bone CT, although cases with narrow IACs, measuring less than $1.5 \mathrm{~mm}$, may be more frequently associated with the absence of the CVN. Despite the fact that, MRI findings were often correlated with the surgical findings regarding the presence or absence of the CVN, a very thin CVN identified during ABI operation was not detectable on MRI of one patient. Promontory EABR failed to show any consistent response in any of the patients. Despite the lack of response on promontory EABR in any of these patients, a CVN was identified during ABI surgery in four patients. Although intracochlear EABR is considered to be more precise than promontory stimulation, it also bears certain problems of its own. Intracochlear EABR was shown to have limitations in precisely predicting the presence or absence of the CVN in this study, too. In particular, it was not possible to acquire any auditory response in a patient due to artifacts induced by muscle potentials resulting from stimulation of the facial nerve.

Song et al. [8] finally concluded that residual response on pure tone audiometry and behavioral response to environmental sounds appeared to be more accurate markers for predicting the presence or absence of the CVN compared to imaging or electrophysiologic testing because all three patients who showed a response to sound stimuli demonstrated thin CVNs during surgery. Our team also reached to a similar conclusion that is, audiological tests seemed to be more important in decision making between $\mathrm{CI}$ and $\mathrm{ABI}$. However, in our series, there are a few patients who showed certain progress initially with CI, but could not carry on when more sophisticated learning processes were required.

As can be seen, it is still a problematic issue to decide between $\mathrm{CI}$ and $\mathrm{ABI}$ in patients with narrow IAC and hypoplastic nerve. Intracochlear eABR might be a better indicator compared to preoperative electrophysiological tests.

\section{Members of ABI Team}

ABI surgery is a technically demanding operation. The team has to be experienced on the surgery, audiological follow up and rehabilitation of CI patients. In addition, an experienced pediatric neurosurgeon is indispensable to achieve success and also to avoid possible complications as much as possible. If the surgery leads to cranial nerve damage and/or brainstem injury which brings forth neurological sequels in otherwise healthy children, this would be a catastrophe both for family and the team. Besides, this might create negative impact on public opinion regarding ABI surgery. It is very important to avoid any possible complications in these children by working with an appropriate team. Placing the implant in the brainstem involves the close collaboration of an experienced pediatric neurosurgeon and pediatric anesthesiologist together with the otologist who is experienced in implant surgery. Occasionally, location of the lateral recess is not apparent and careful dissection is necessary to identify the exact location. This is one of the most important issues to prevent malposition of the electrode which may lead to unsuccessful results and an experienced neurosurgeon is the key to avoid this complication.

\section{The Age Limit For ABI in Children}

According to the consensus statement age limit for $\mathrm{ABI}$ in children is similar to CI patients [2]. Better language outcome is expected when the children are operated between 1 and 2 years of age. ABI surgery is more challenging than CI surgery because, young children have less blood volume and cerebrospinal fluid (CSF) in the posterior fossa. From the neurosurgical point of view, in the consensus paper optimum lower limit was determined as 18 months but, depending on the experience of the center, but it was also suggested that it may be done as early as 12 months old. 
Early intervention will have better audiological outcome. Although the surgical risks will be less when the child is operated later on in their life, the language outcome will not be satisfactory because of the brain plasticity. This will lead to discredit the surgery as it will be thought that this intervention will not produce good hearing and language outcome. Therefore, ideal age appears to be between 1 and 3 years of age. As these are prelingually deafened children, this procedure should not be offered to patients older than 5 years old.

\section{Preoperative Evaluation}

All members of the team has to evaluate ABI candidates in detail.

Radiological workup involves HRCT and MRI of the temporal bone. Classification of the malformation can be done better with HRCT. Diagnosis and indication for ABI is straight forward with HRCT in cases such as Michel deformity and cochlear aplasia. Children with cochlear hypoplasia, hypoplastic cochlear aperture, and narrow IAC need more careful audiological and radiological evaluation with MRI. MRI on the other hand demonstrates the neural structures in the IAC. Any vascular abnormality around the lateral recess can be seen on MRI. Side with more developed inner ear or the CVN should be preferred. As stated in the preceding paragraphs MRI has limitations in the diagnosis.

Side selection is very important in ABI surgery. The team should try to choose the side where more information can be provided to the cochlear nucleus. Therefore, side with more developed neural structures (e.g., facial nerve presenting unilaterally, or more prominent CVN or vestibular nerve may imply better developed cochlear nucleus area). If equal under all conditions, more developed inner ear (if there is a cochlear aplasia on one side and a hypoplastic cochlea on the other side, the latter can be preferred). In addition, side where the entrance of the lateral recess is more favorable, and the lateral recess is more accessible (where cerebellar retraction will be less) can be chosen.

\section{Audiological Assessment Procedure}

For preoperative evaluation of ABI candidates, all audiological test battery should be applied. This test battery includes both subjective and objective tests. It is apparent that in patients with complete labyrinthine aplasia and cochlear aplasia no response is expected. But even in these patients sometimes with maximum audiometric limits some response is observed in low frequencies which is in accordance with tactile sensation.

In subjective tests, the candidate should be evaluated with insert phones and if not possible, free field evaluation should be done. According to the age of the child, behavioral observation audiometry (BOA), visual reinforced audiometry, or play audiometry can be used.

For objective evaluation, it is appropriate to start with tympanometry and acoustic reflex tests to show middle ear status for all age groups, especially for infants and children. These tests should be followed by otoacoustic emissions (OAEs), and auditory brainstem response (ABR) measurements.

Subjective tests are very important even when no response is obtained by other tests, including the objective ones. In this situation subjective tests are the only method which give information about hearing status of the patient. Some patients with hypoplastic cochlear nerve demonstrate behavioural response with pure tone or speech stimulation. These patients are counselled that the ear with best response with insert phone will be selected for CI, and the patient will be followed up for 6-9 months with CI. At the end of this period an eABR is also done to see if there is any response with CI. If there is no development in speech perception and no response on eABR, ABI will be offered to the family. It is also very important to take into consideration the observation of the family. In this situation we choose the opposite ear for ABI, thereby providing bilateral amplification to these children.

\section{Rehabilitative Assessment Procedure}

A detailed medical history is taken from patient's family. Anamnesis includes chronological age of child, age of awareness of deafness, and age of diagnosis. Information about school, therapy, additional handicaps, and current medical condition is also obtained. Based on this information family structure and social support systems are evaluated.

Next step is assessment of child's auditory perception skills, language and speech skills and learning abilities. Auditory perception skills are evaluated with Ling's Six Sound Test and IT-MAIS/MAIS. According to the child's auditory performance, pattern perception tests, word recognition tests and auditory comprehension tests are applied.

Language development is assessed and receptive and expressive language development level is determined. On the other hand more information about child's daily communication methods (sign, auditory-verbal), vocabulary capacity etc. is obtained from the family. Besides auditory and language development, general developmental milestones are evaluated by screening tests and taking history from parents. Lastly all the information is reviewed with parents. Opinion of the family about ABI is asked and rehabilitation process after surgery is explained. If family needs psychological support or child needs psychological evaluation, psychological consultation is offered. 
As in the evaluation period of CI candidates, neurological evaluation should also be done if necessary.

\section{Surgery}

ABI surgery can be performed through translabyrinthine, retrosigmoid or retrolabyrinthine approach [9••]. In children main approach for auditory brainstem implantation (ABI) has been retrosigmoid approach. Temporal bone is much smaller in a child of 2-3 years of age when compared to an adult. As a result translabyrinthine approach will provide much more limited surgical exposure than retrosigmoid approach in a child. In addition, drilling of the temporal bone takes more time to expose the brainstem in comparison to retrosigmoid approach. Therefore, for the placement of ABI in a child, retrosigmoid approach appears to be advantageous. In addition, retrosigmoid approach makes it possible to bypass the mastoid air cells so that intracranial contamination by the middle ear flora can be prevented. The landmarks, advantages and disadvantages of different approaches have been discussed in detail previously in another paper regarding ABI surgery in children $[9 \bullet \bullet]$.

However, translabyrinthine approach has been utilized for ABI in a child by Helge Rask Andersen and his team (not published, personal communication) and the electrode was successfully placed into the recess.

Bento et al. [10] described the extended retrolabyrinthine approach (RLA) for ABI placement which was performed consecutively in three children without any further complications. They stressed the importance of radiological examination both in evaluation of the etiology and to choose the side to be operated on for RLA based on the size of the jugular bulb. They advised that, side with less prominent jugular bulb should be chosen. They stated that approach is more familiar to the otologist. After a postauricular incision and mastoidectomy, they identified jugular bulb as the main landmark for access to the dura. It was exposed by removing bone from its entire circumference. Only the intracranial portions of the seventh and eighth cranial nerves were exposed. Then cerebellar flocculus and lower cranial nerves were identified. After retracting the choroid plexus they identified foramen of Luschka and placed the ABI electrode. RLA was chosen due to their extensive experience in using this technique for vestibular schwannoma surgery in patients with useful hearing. RLA allowed direct visualization of the foramen of Luschka through a limited approach. There was no requirement for cerebellar retraction or even for opening the internal auditory meatus and semicircular canals. The disadvantage of this approach in children is that it cannot be used in a very young child with an extremely large jugular bulb.
As a result, all three approaches can be used in ABI surgery of children but retrosigmoid approach is still being the most widely used technique when compared to the other two methods. With any preferred method, it should be noticed that distorted anatomy at the cerebellopontine angle, at the cranial nerve entry zones and brainstem due to absence of the CVN makes surgery more difficult at certain cases [1].

\section{Intraoperative Monitoring}

After placement of the electrode, electrical ABR is utilized to identify the localization of the cochlear nucleus. Different electrodes and electrode groups are stimulated one by one to check the position of the ABI electrode in relation to the cochlear nucleus. This will help to position the electrode array to maximize auditory stimulation while nonauditory stimulation is minimized. In children, the recess is not very large therefore, after placement, usually only slight lateral movements are possible. If the electrode is too deeply inserted, there will be response only on the lateral channels. This necessitates minimal pulling out of the electrode. Similarly if the response can only be obtained from the channels localized at the tip of the plate electrode, it should be slightly inserted deeper into the recess. In adults, senior author (LS) encountered a few cases where the width of the lateral recess was twice the size of electrode. In these cases the electrical ABR is very useful in confirming placement of the array. Slight adjustments in the position of the array should be made according to electrical auditory responses. The surgeon and the audiologist should be familiar with the numbers of individual active channels on the electrode array. A diagram showing the channels for both left and right sides should be kept in the operating room to avoid confusion about electrode orientation. Position of an individual active channel of an already inserted electrode on the left side is completely opposite on the right side.

In patients undergoing $\mathrm{ABI}$ surgery, an intraoperative eABR demonstrating III. and V. waves is a valuable finding (Fig. 7a). This shows that the electrode is in the correct location. Sometimes there may be no response (Fig. 7b) or myogenic activity (Fig. 7c). Myogenic activity shows a possible future side effect. In this situation the position of the electrode array is adjusted according to the findings.

\section{Initial Stimulation and Follow Up}

The first programming of the ABI electrode is done 5-6 weeks after the surgery. For the first 4 patients we waited for 3 months after the surgery, but now the device is 
Fig. 7 Intraoperative eABR findings. eABR images showing the III and Vth waves (a), no response (b), and myogenic activity indicating a future possible side effect (c) (a)

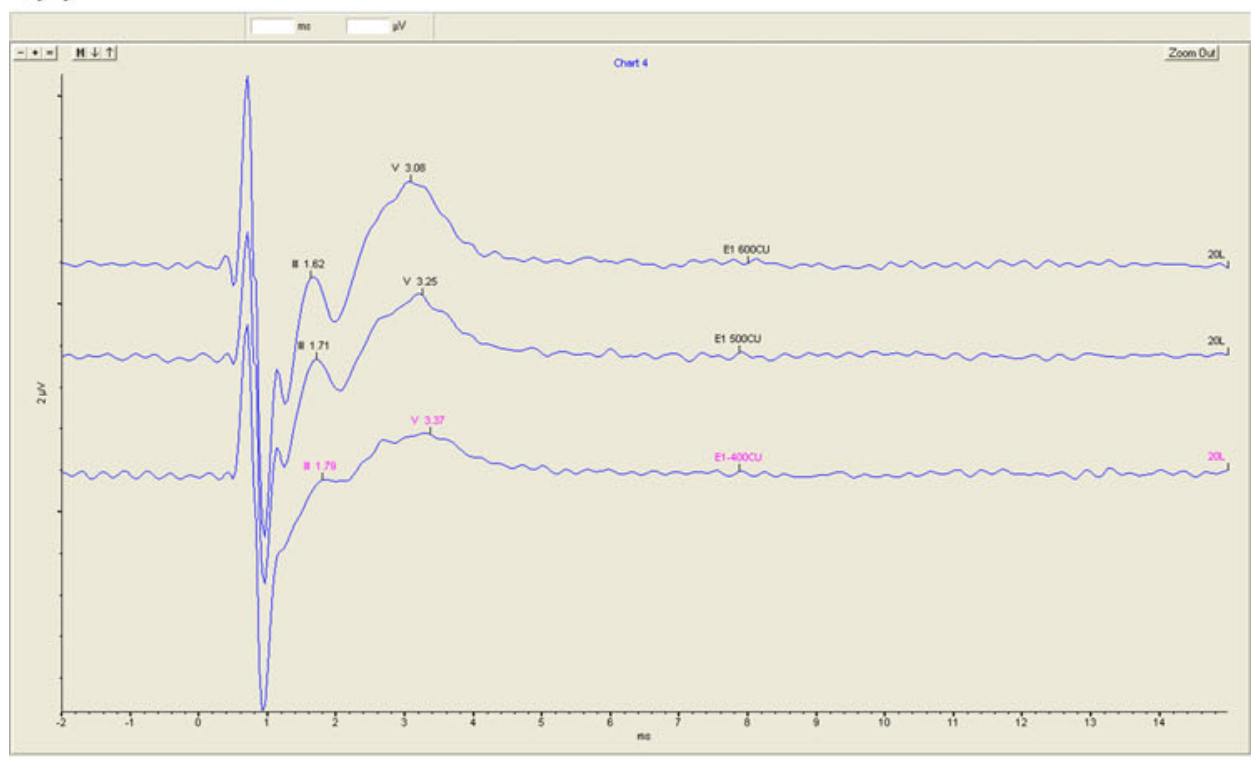

(b)

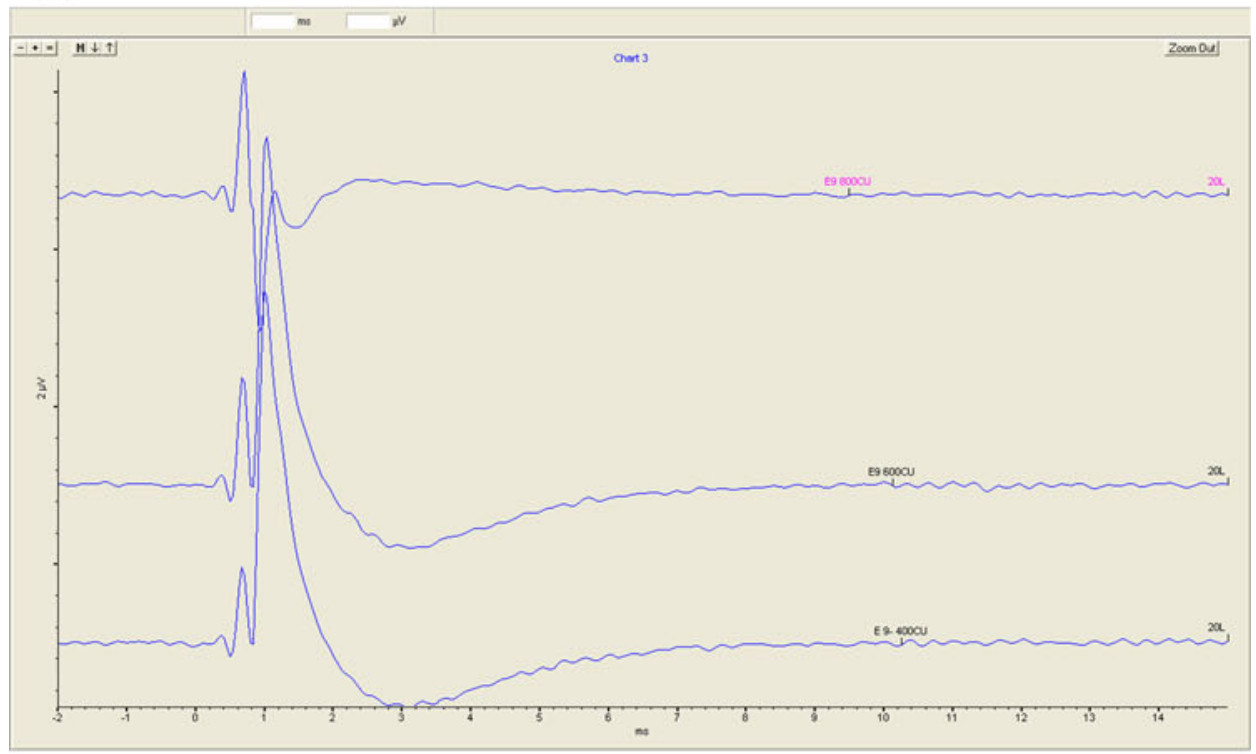

(c)

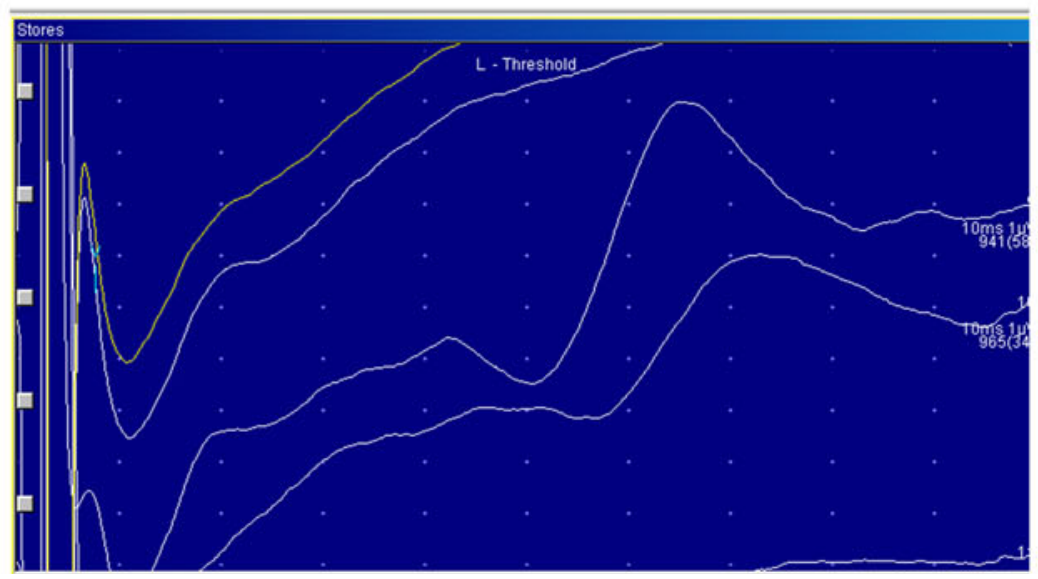


stimulated 5-6 weeks after the surgery. General anesthesia is not required; monitoring the child is sufficient.

Most-comfortable levels (MCLs) are found by increasing the current level step by step. During this time behavioral responses and side effects are observed. After MCLs are determined, all MCLs are decreased by 5 or 10 current unit (CU), and speech processor is activated. This decrement is done because the integrated level of all channels can be annoyingly loud for the first stimulation.

Initially, the channels in the center of the electrode are activated. If there are no side effects, then it is possible to proceed to neighboring ones. Usually 6-7 channels are activated in the first visit. The rest of the channels are activated during the second visit which occurs usually 1 month after initial programming. If there is a side effect, the current level is lowered until hearing sensation without any side effects is achieved. If this is not possible, the channel leading to the side effect is closed. A few months later, the channel(s) causing side effects are activated once again. It has been observed that in many occasions, the channels initially causing side effects start to produce only auditory stimulation without any adverse reaction (Fig. 8a, b). The ones prompting side effects can be kept closed permanently.

Fitting infants and young children is a complex work, due to fact that no adult-like clear responses can be obtained from them. In most of the cases they perform some behaviors with sound stimulation. These may be cessation of activity, looking at mother, holding or showing the implant side or crying. These programing sessions must be done by experienced pediatric audiologists. Side effects must be observed and monitored particularly during the first stimulation. These can vary from single cough, to stimulation of vagus nerve which organizes hearth beat. So it is essential to perform this section in the presence of a
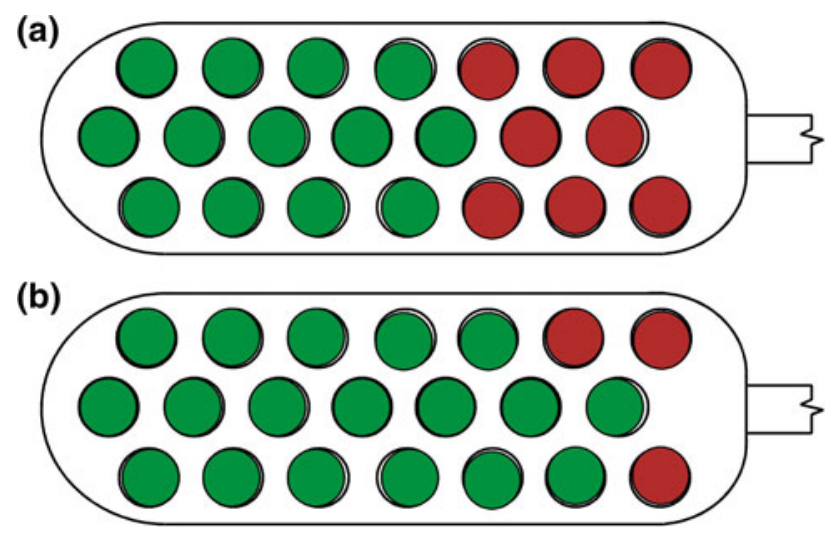

Fig. 8 Postoperative distribution of channels. a showing auditory stimulating (green) and no response-side effects (red) channels of an electrode. b Majority of the patients demonstrate increase in the number of auditory stimulating channels over time (Color figure online) medical doctor in case of cardiac arrhythmia. The initial program gives very important information for follow up. These are all noted for future programming.

In Hacettepe University we have done eABR before initially stimulation for the first patients. It has been observed that this does not add more information than the intraoperative eABR measurements. Today eABR is not performed anymore. We use intraoperative findings for the first programing section.

\section{Audiological Outcome}

Until now there are limited number of articles regarding the outcome of ABI in children. Although, prognostic factors are not clear enough, it is obvious that surgery at younger age affects outcomes positively enabling higher neuronal plasticity, as it is well known in CI patients.

Colletti [11] reported the initial auditory results of ABI in children. All of the 14 prelingually deafened children had environmental sound awareness, detection of instrumental sounds and lip-reading enhancement. Three prelingual children achieved bisyllabic word recognition as well understanding of simple commands. When their article was written, none of those children had open set recognition. They speculated that the activation of the ABI, in children with no cochlear nerve, can facilitate the development of some cognitive parameters related to selective visual- spatial attention and fluid (multisensory) reasoning (repeated patterns subtest), as demonstrated by the fact that these two subtests increased significantly over the 1-year period following switch-on in all seven children.

Eisenberg et al. [12] reported the result of a 3-year-old child who received an ABI at the age of two. After 12 months of ABI use the child was able to identify speech patterns consistently, with developing closed-set word identification. Language age with signs was approximately 2 years, and vocalizations were increasing. Of normal intelligence, he exhibited attention deficits with difficulty in completing structured tasks. They concluded that his scores were at the median of the range shown for a small sample of CI children implanted at a similar age. Also, they agreed with consensus regarding timing of ABI surgery, i.e. children younger than 3 years of age would be better candidates for ABI rather than older children.

Goffi-Gomez et al. [13] reported their ABI experience in four children. Three of them had cochlear malformation and one ossified cochlea. All of the patients were regular users of the device, with more than $6 \mathrm{~h}$ a day of implant use. Two were 3 years old and one was 7 years old. They had 8,9 and 10 active number of electrodes. They all showed some improvement in their auditory skills which was demonstrated by their postoperative tests. All received 
oral rehabilitation and had a slight improvement in the ITMAIS/MAIS scores. In this group, as can be seen, one patient is a 7 year old which brings considerable difficulty about obtaining satisfactory language development. Despite lack of good language development, all children use their devices regularly their ABIs.

According to Goffi-Gomez et al. [13] Diamante and Pallares reported two children implanted with ABI who were 3 and 10 years old. Both achieved audiograms with $35 \mathrm{dBHL}$, had full detection of Ling sounds and responded to their names at 6 months after the operation.

Choi et al. [14] reported their experience in nontumor patients. All eight patients with narrow IACs clearly expressed behavioral responses following initial stimulation using an ABI. All of the patients demonstrated an improvement in auditory performance with time. As in CI, additional handicaps such as mental retardation and blindness were associated with delayed development of auditory function after ABI. They concluded that this can be attributed to problems with higher cognitive function rather than influences of the auditory stimulation itself. Six of their patients had had no sound perception after CI for a mean period of 5 years approximately. This rather long waiting period urged the authors for an earlier intervention so as to obtain better speech development. They started to use intracochlear EABR or functional MRI to assist in determining the appropriate timing of ABI. In their institution they started to counsel the parents to perform CI initially and to plan further rehabilitation according to the results of intracochlear EABR performed intraoperatively or during the immediate postoperative period. In case of no response with intracochlear EABR, ABI was recommended in earlier period following CI, whereas patients demonstrating satisfactory responses on intracochlear EABR were rehabilitated with a CI for a longer period of time.

We also reported preliminary results of 11 children with severe labyrinthine anomalies and had ABI between 2006 and 2008 [15]. We used retrosigmoid approach in all patients. Six children gained basic audiologic functions and were able to recognize and discriminate sounds, and many could identify environmental sounds such as a doorbell and telephone ring by the third month of surgery. Improvement in mean performance on Meaningful Auditory Integration Scale was apparent for all operated children. Improvement in Meaningful Use of Speech Scale scores of 2 patients, demonstrating that the child using its own voice for speech performance, was observed between the baseline and 12th month. At the beginning, 5 children were able to identify Ling's 6 sound by the end of 2-6 months, and 2 of them also started to identify words due to their pattern differences and multisyllabic word identification by $6-9$ months. Two children with attention deficit hyperactivity disorder have made slower progress than the other children with ABIs.
Until January 2013, 39 children with severe inner ear malformations received ABI in Hacettepe University. 29 of these children have been followed for more than 1.5 years of ABI use. All 29 children use their devices regularly on daily basis. 25 of 29 patients ( $86 \%$ ) detected all of Ling's sounds; 22 of 29 patients ( $75 \%$ ) recognized all sounds in the test; 18 (64\%) have MAIS scores between 30 and 40 . With Daily Sentence Test in Turkish, 10 of the patients have scores between 60 and $100 \%$ (auditory-verbal) and 8 have scores between 20 and $100 \%$ (only auditory).

Interindividual variability in language development is due to chronological age, duration of ABI use, additional handicaps and cognitive development. The most important factor appears to be the associated comorbidity due to additional handicaps such as attention deficit hyperactivity, slight mental retardation, visual problems, etc. Majority of the patients with limited improvement in performance have additional handicaps. Young children who started to use ABI earlier showed better language performance. Almost all patients had some slight, acceptable side effects due to electrical stimulation of the brainstem. However, none of them had any major issues such as cardiac problems so far.

In literature, there is very limited data regarding results of ABI application in children with postmeningitic cochlear ossification. Goffi-Gomez et al. [13] mentioned a child who was implanted with ABI at 38 months of age due to meningitic cochlear ossification. They reported that, audiological outcome was poor in that child after 1 year follow-up. Colletti and Zoccante [16] also had one child with $\mathrm{ABI}$ who was postmeningitic in their group of patients. Although they did not state outcomes of that particular case separately, overall perceptual performance was considered to be satisfactory in all children. It is obvious that, more experience and accumulation of results are required in order to reach certain conclusions for this special group of children.

\section{Surgical Complications}

Colletti et al. [17] reported the complications of ABI surgery in their series composed of adults and children. They had no mortality. One child had a slow recovery after surgery, a computed tomographic scan revealed an intracerebellar clot. Revision surgery was performed, and clot was evacuated. He had a full neurologic recovery. Another child developed meningitis. This resolved uneventfully with medical treatment. As a minor complication they observed temporary asymptomatic cerebellar edema in the postoperative computed tomographic scans in 9 children. They were all treated successfully with steroids and diuretics. Four children developed postoperative wound seroma which was successfully treated with aspiration and 
pressure dressing. Apart from these, infection of the incision, temporary dysphonia and balance disorders occurred in certain patients but resolved after treatment. The authors concluded that the surgery bares less complications when compared to ABI operation of NF2 patients and overall complication rate of $\mathrm{ABI}$ is not much greater than that of $\mathrm{CI}$ and comparable to neurovascular decompression.

Bayazit et al. [18] reported two cases of postoperative CSF leakage following ABI surgery in five children. Attention was drawn to possible long term complications such as device failure, infection, biofilm formation or extrusion, about which still knowledge is limited.

In our series of children, one of the initial 3 patients had postoperative rhinorrhea. He was revised immediately and the defect in the mastoid was repaired. Also, 2 patients had transient facial nerve palsy which resolved completely within 2 weeks. This was attributed to the cerebellar retraction. In one patient, severe cerebellar edema occurred intraoperatively which impeded rest of the surgery. Therefore, operation was stopped and completed in the second session uneventfully 3 weeks later. Seroma occurred in five patients due to CSF leakage. In four patients it was easily controlled in a few days, with lumbar drainage and serial dressings. However, in one patient, CSF leak continued despite these measures and prolonged the hospitalization period markedly. None of our patients had to be revised due to seroma; mentioned conservative treatment was successful enough to manage this complication. In these patients CSF leakage was thought to occur around the electrode lead from subarachnoid space to subcutaneous tissue. It is important to place pieces of soft tissue around the electrode at the level of dura in order to attain effective sealing and lumbar drainage is used now routinely to avoid CSF leakage. Both of these measures were successful and this complication was not experienced in the rest of the group.

Overall results showed that this procedure can be performed with minimum risks in centers with experienced otology, neurosurgery and anesthesia facilities.

\section{Conclusion}

ABI in children provides auditory sensation when properly placed into lateral recess. Side effects due to the stimulation of the neighboring cranial nerves are common which can be overcome by decreasing current level or closing the channel permanently. Every effort should be shown to decrease the intracranial complications by working in collaboration with an experienced otologist, pediatric neurosurgeon and anesthesiologist. Satisfactory audiological outcome with language development is possible but handicaps impede success of outcomes. Probable indications still continue to be challenge for the implant team.
Disclosure No potential conflicts of interest relevant to this article were reported.

\section{References}

Papers of particular interest, published recently, have been highlighted as:

- Of importance

•- Of major importance

1. Colletti V, Fiorino F, Sacchetto L, et al. Hearing habilitation with auditory brainstem implantation in two children with cochlear nerve aplasia. Int J Pediatr Otorhinolaryngol. 2001;60(2):99-111.

2. Sennaroglu L, Colletti V, Manrique M, et al. Auditory brainstem implantation in children and non-neurofibromatosis type 2 patients: a consensus statement. Otol Neurotol. 2011;32(2): 187-91.

3. - Sennaroglu L. Cochlear implantation in inner ear malformations-a review article. Cochlear Implants Int. 2010;11(1):4-41. This review article includes classification of inner ear malformations; understanding of which is very important in determining indications of $A B I$ in children.

4. Warren FM 3rd, Wiggins RH 3rd, Pitt C, et al. Apparent cochlear nerve aplasia: to implant or not to implant? Otol Neurotol. 2010;31(7):1088-94.

5. • Valero J, Blaser S, Papsin BC, et al. Electrophysiologic and behavioral outcomes of cochlear implantation in children with auditory nerve hypoplasia. Ear Hear 2012;33(1): 3-18. Auditory nerve hypoplasia cases constitute the most challenging group in decision making between $C I$ and ABI. This report focuses on objective audiological evaluation methods and their correlation with radiological findings.

6. Buchman CA, Teagle HF, Roush PA, et al. Cochlear implantation in children with labyrinthine anomalies and cochlear nerve deficiency: implications for auditory brainstem implantation. Laryngoscope. 2011;121(9):1979-88.

7. Song MH, Bae MR, Kim HN, et al. Value of intracochlear electrically evoked auditory brainstem response after cochlear implantation in patients with narrow internal auditory canal. Laryngoscope. 2010;120(8):1625-31.

8. Song MH, Kim SC, Kim J, et al. The cochleovestibular nerve identified during auditory brainstem implantation in patients with narrow internal auditory canals: can preoperative evaluation predict cochleovestibular nerve deficiency? Laryngoscope. 2011; 121(8):1773-9.

9. - Sennaroglu L and Ziyal I. Auditory brainstem implantation. Auris, Nasus, Larynx 2012;39(5): 439-50. The review article is advised to be read in order to obtain basic information regarding ABI.

10. Bento RF, Monteiro TA, Tsuji RK, et al. Retrolabyrinthine approach for surgical placement of auditory brainstem implants in children. Acta Otolaryngol. 2012;132(5):462-6.

11. Colletti L. Beneficial auditory and cognitive effects of auditory brainstem implantation in children. Acta Otolaryngol. 2007; 127(9):943-6.

12. Eisenberg LS, Johnson KC, Martinez AS, et al. Comprehensive evaluation of a child with an auditory brainstem implant. Otol Neurotol. 2008;29(2):251-7.

13. Goffi-Gomez MV, Magalhães AT, Brito Neto R, et al. Auditory brainstem implant outcomes and MAP parameters: report of experiences in adults and children. Int J Pediatr Otorhinolaryngol. 2012;76(2):257-64. 
14. Choi JY, Song MH, Jeon JH, et al. Early surgical results of auditory brainstem implantation in nontumor patients. Laryngoscope. 2011;121(12):2610-8.

15. Sennaroglu L, Ziyal I, Atas A, et al. Preliminary results of auditory brainstem implantation in prelingually deaf children with inner ear malformations including severe stenosis of the cochlear aperture and aplasia of the cochlear nerve. Otol Neurotol. 2009;30(6):708-15.

16. Colletti L, Zoccante L. Nonverbal cognitive abilities and auditory performance in children fitted with auditory brainstem implants: preliminary report. Laryngoscope. 2008;118(8):1443-8.
17. Colletti V, Shannon RV, Carner M, et al. Complications in auditory brainstem implant surgery in adults and children. Otology \& neurotology : official publication of the American Otological Society, American Neurotology Society [and] European Academy of. Otology and Neurotology. 2010;31(4):558-64.

18. Bayazit YA, Abaday A, Dogulu F, Göksu N. Complications of pediatric auditory brain stem implantation via retrosigmoid approach. ORL J oto-rhino-laryngology Relat Spec. 2011;73(2): $72-5$. 